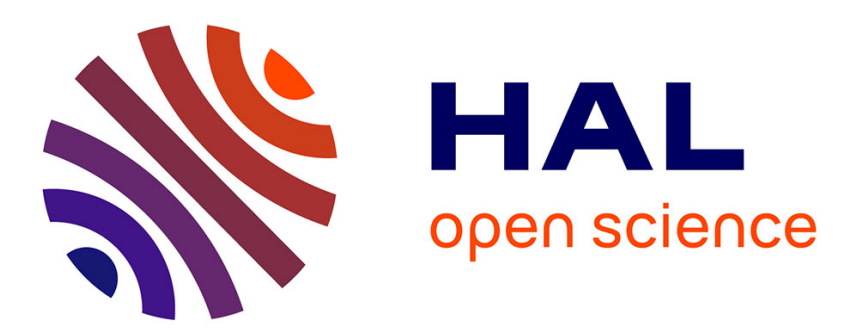

\title{
Politiques de codéveloppement et le champ associatif immigré africain : un panorama européen
}

Thomas Lacroix

\section{To cite this version:}

Thomas Lacroix. Politiques de codéveloppement et le champ associatif immigré africain : un panorama européen. African Yearbook of International Law / Annuaire Africain de droit international, 2010, 2008-16, pp.79-98. halshs-00820729

\section{HAL Id: halshs-00820729 \\ https://shs.hal.science/halshs-00820729}

Submitted on 27 Jan 2014

HAL is a multi-disciplinary open access archive for the deposit and dissemination of scientific research documents, whether they are published or not. The documents may come from teaching and research institutions in France or abroad, or from public or private research centers.
L'archive ouverte pluridisciplinaire HAL, est destinée au dépôt et à la diffusion de documents scientifiques de niveau recherche, publiés ou non, émanant des établissements d'enseignement et de recherche français ou étrangers, des laboratoires publics ou privés. 


\section{POLITIQUES DE CODEVELOPPEMENT ET LE CHAMP ASSOCIATIF IMMIGRE AFRICAIN : UN PANORAMA EUROPEEN}

\section{Thomas Lacroix*}

\section{Introduction}

Les politiques s'appuyant sur les migrations comme instrument de développement sont loin d'être une nouveauté. Cette thématique apparaît et disparaît de l'agenda des décideurs de façon récurrente depuis quarante ans en fonction $\mathrm{du}$ contexte économique ${ }^{1}$. Les organisations internationales telles que la Banque Mondiale, l'Organisation des Migrations Internationales ou l'ONU sont les principaux acteurs du renouveau de cette réflexion. Des rencontres internationales telles que le Dialogue de Haut Niveau sur les Migrations et le Développement, organisé à l'occasion de la $61^{\text {ème }}$ session de l'Assemblée Générale de l'ONU (New York, 2006,), le Forum Mondial sur la Migration et le Développement (Bruxelles, 2007 ; Manille, 2008; Athènes, 2009), la conférence de Bellagio (Turin, 2007) et d'autres événements, ont généralisé l'intérêt pour la question. L'engouement récent qui a marqué cette dernière décennie a lui-même de multiples raisons. En lien avec la croissance des transferts des migrants depuis la fin des années 2000 (252 milliards de dollars en 2007 selon la Banque Mondiale), il est de plus en plus admis que la migration peut être un instrument de développement des pays de départ. Toutefois, à l'échelle nationale et européenne, le

\footnotetext{
Chercheur, International Migration Institute, University of Oxford.

1 De Tapia S., Système migratoire euroméditerranéen, Strasbourg: Editions du Conseil de l'Europe, 2008,130p.
} 
codéveloppement apparaît davantage comme un moyen de consolider le contrôle des flux migratoires. Enfin, à l'échelon local, cette politique est davantage liée à une nouvelle approche de l'intégration.

Le codéveloppement peut donc être appréhendé sous de multiples aspects : comme une stratégie de développement, de maîtrise de flux ou d'intégration, voire sous l'angle des tractations diplomatiques qu'elle engendre. C'est toutefois la société civile africaine en Europe qui a été le plus affecté par ces nouveaux dispositifs. Les populations africaines en Europe ont été les premières ciblées par ces dispositifs. Les organisations africaines en Europe ont ainsi trouvé un nouveau terrain de visibilité dans l'espace public.

L'objectif de cet article est donc double. Nous aborderons les différentes approches de la politique de codéveloppement en Europe. Nous traiterons de l'articulation de ces politiques aux niveaux national et local dans les pays d'accueil et montrerons en quoi le sens de ces politiques diffère à ces deux niveaux. Le second objectif de cet article sera de tracer les contours de l'impact de ces dispositifs sur la société civile migrante. Nous présentons ici successivement l'évolution des politiques de codéveloppement initiées par la Grande-Bretagne, la France, l'Italie et l'Espagne. Nous distinguons ici le cas de la GrandeBretagne qui tente d'intégrer la migration dans sa politique de coopération au développement et l'Espagne, l'Italie et la France qui ont explicitement adopté le codéveloppement dans une optique de gestion des flux migratoires.

\section{Grande-Bretagne : migration et lutte contre la pauvreté}

La lutte contre la pauvreté est devenue le mot d'ordre de la politique de coopération britannique lors de la prise de pouvoir des travaillistes en 1997. DFID (Department for International Development), le Ministère de la coopération britannique, a publié une série de livres blancs sur la question : Eliminating World Poverty: A Challenge for the $21^{\text {st }}$ Century $(1997)^{2}$, Getting It Right Together (2001), Moving out of Poverty: Making Migration Work for Poor

2 DFID, Eliminating World Poverty: A Challenge for the $21^{\text {st }}$ Century. Livre Blanc, Department for International Development, Londres, 1997. 
People $(2007)^{3}$. Ces documents placent les migrations au premier rang des instruments de lutte contre la pauvreté dans les pays du Sud. Le livre blanc publié en 2007, précédé de la conférence de Londres intitulée Making Migration Work for the Developing World (juin 2007), précise les grandes lignes autour desquelles s'articule la stratégie de DFID en la matière. Il met en évidence l'importance de distinguer les différents types de migration, avec un accent particulier pour les migrations forcées, les femmes et enfants et les migrations Sud/Sud qui constituent des catégories particulièrement liées aux phénomènes de pauvreté. Cette politique s'articule autour de deux axes principaux : l'inscription des organisations de migrants dans la politique de coopération et la facilitation des transferts migratoires. Nous présentons ici les deux axes de cette politique.

Dès 1997, la politique de coopération de DFID intègre le savoirfaire des migrants comme un instrument de développement ${ }^{4}$. Mais il faut attendre 1999 pour que se mette en place une consultation des acteurs du développement incluant un sous-groupe d'organisations de migrants, le DAWG (Development Awareness Working Group). Le décalage entre les attentes de ces acteurs et les objectifs des pouvoirs publics ont poussé DFID à financer la création de Connection for Development (CfD), une plateforme regroupant les associations de migrants impliquées dans le développement de leur pays d'origine. En avril 2003, est voté un plan de financement de trois ans, le Strategic Grant Agreement (SGA), à hauteur de $750000 £$. L'objectif du CfD est principalement de sensibiliser les organisations de migrants à la question du développement (seule une minorité d'entre elles est active dans ce domaine), et de leur fournir la possibilité d'accéder aux sources de financement. Ces efforts n'ont eu qu'un succès limité puisque les organisations de migrants demeurent largement minoritaires parmi les candidats aux financements de la coopération $^{5}$. Le SGA ne fournit cependant pas de financement pour

3 DFID, Moving of Poverty: Making Migration Work Better for People, Department for International Development, Londres, 2007.

4 [To] Build on the skills and talents of migrants and other members of ethnic minorities within the UK to promote the development of their countries of origin, DFID, Eliminating World Poverty: A Challenge for the $21^{\text {st }}$ Century. Livre Blanc, Department for International Development, Londres, 1997.

5 De Haas H., Engaging Diasporas. How Governments and Development Agencies 
les projets au Sud. Le SGA n'ayant pas été renouvelé en 2009, le CfD a cessé ses activités. En dehors du CfD, DFID a également intégré des organisations diasporiques dans une série de Plans d'Action Pays (Country Action Plan) avec le Bangladesh, le Pakistan, le Nigeria et $1^{\prime}$ Inde $^{6}$. Enfin, dans le domaine des migrations qualifiées et de l'investissement productif, DFID a soutenu l'initiative AfricaRecruit ${ }^{7}$ depuis 2002, lancée par le NEPAD (Nouveau Partenariat pour le Développement de l'Afrique) et le Commonwealth.

Sur les 404 associations enregistrées en 2007 dans la plateforme du $\mathrm{CfD}$, le groupe le plus important était celui des organisations africaines (162, soit $40 \%$ des effectifs). La politique de mobilisation des diasporas pour le développement a contribué à faire émerger des organisations influentes dans le champ de la coopération. La plus importante est AFFORD (African Foundation For Development), organisation active dans la promotion du rôle des ONG de migrants. Elle a, dans cette optique, publié un rapport dans le cadre du Dialogue de Haut Niveau de l'ONU sur les Migrations et le Développement en $2006^{8}$, ainsi qu'un commentaire dans le cadre de la préparation du livre blanc de DFID en $2000^{9}$. Le versant de leurs activités en Afrique est essentiellement axé sur l'Afrique de l'Ouest, au Ghana et en Sierra Leone. Le projet principal, SEEDA (Supporting enterprise and entrepreneurship for Africa's development) est destiné à soutenir la création d'entreprise en s'appuyant sur les transferts de compétence des émigrés. La dualité du travail de AFFORD (à la fois groupe de pression au niveau politique et acteur de terrain) est caractéristique de la nouvelle génération d'ONG de migrants apparue dans le sillage des politiques associant migration et développement.

Le second versant de la politique britannique vise à faciliter les

Can Support Diaspora Involvement in the Development of Their Origin Countries, International Migration Institute (IMI), University of Oxford, for OXFAM NOVIB, 2006, p. 62.

Idem.

Voir le site Internet (www.africarecruit.com).

8 Chuku-Emeka C., Report on United Nations High-level Dialogue in International Migration and Development, African Foundation for Development, Londres, 2006.

9 AFFORD, Globalisation and Development: A Diaspora Dimension, Department for International Development, Londres, 2000. 
transferts vers les pays d'origine. Un groupe de travail sur les transferts a permis la mise en place d'un site internet destiné à fournir aux migrants les informations pour transférer de l'argent au meilleur coût ${ }^{10}$. En 2004, les migrants résidant en Grande-Bretagne ont envoyé environ 2,3 milliards de livres sterling ${ }^{11}$. A l'échelle internationale, DFID est partie prenante de différents processus engagés avec des Etats du Sud (Remittance Country Partnership, incluent le Ghana, Nigeria et Bangladesh), des organisations internationales (InterAgency Remittances Task Force, Remittances Principles Task Force) et partenaires privés telles que les compagnies de transferts (UK Remittance Working Group).

Les débats concernant les relations entre migration et développement se déroulent essentiellement au niveau du Ministère de la coopération. Le Ministère des Affaires Etrangères et surtout le Ministère de l'Intérieur semblent n'avoir été inclus que de façon marginale dans ces discussions. Le fait que la politique d'entrée et de séjour des étrangers soit totalement déconnectée de la prise en compte des impacts sur le développement est révélateur de ce fossé. Cela s'est traduit par une politique d'attraction des étudiants étrangers. Le Royaume-Uni serait ainsi le quatrième pays en termes de diplômés étrangers en science et ingénierie en proportion de sa force de travail (chiffres $\mathrm{OCDE}^{12}$ ) La mise en œuvre récente de la délivrance des visas à points ${ }^{13}$ renforce la sélection des candidats en fonction de leur compétence. La politique migratoire est clairement destinée à satisfaire les besoins en main-d'œuvre de l'économie britannique, sans considération du capital humain des économies des pays de départ. Il en va de même pour la politique d'intégration qui est, depuis les attentats du 11 septembre de New York et du 7 juillet à Londres, guidé par le souci de «cohésion sociale». Plusieurs rapports et documents

10 Voir le site Internet (www.sendmoneyhome.org).

11 Remittance Working Group, UK Remittance Market, Department For International Development, Londres, 2005.

12 Findlay A., Stam A., "International Student Migration to the UK: Training for the Global Economy or Simply Another Form of Global Talent Recruitment?" International Competition for S\&E Students and Workers, Londres, 2006.

13 La délivrance des visas est conditionnée à l'obtention d'un certain nombre de points attribués en fonction de la qualification, l'âge ou la situation familiale et financière des demandeurs. 
stratégiques sur la cohésion sociale ont été publiés depuis cinq ans par les pouvoirs publics. Le lecteur pourra se référer, par exemple, au rapport du Panel sur la Cohésion Communautaire, The End of Parallel Lives, Londres, 2004. Dans ce contexte, les spécificités communautaires et les liens avec le pays d'origine sont jugés suspicieux et potentiellement vecteur de division. La question de la relation entre intégration et codéveloppement est absente des débats britanniques, contrairement à ce qui se passe dans les autres Etats européens.

\section{Les politiques de codéveloppement : France, Espagne et Italie}

Le concept de codéveloppement fut élaboré en France dans les années 1970, au sein des cercles tiers-mondistes du parti socialiste. Mais c'est en 1997, que le rapport Sami Nair donne au terme son acception courante $^{14}$. La pratique du codéveloppement s'est ensuite diffusée en Europe du Sud, dans les nouveaux pays d'immigration. L'Espagne et l'Italie l'ont mis en œuvre essentiellement comme une dimension de la coopération décentralisée.

\subsection{France : entre retour et codéveloppement}

La politique française de codéveloppement en France s'inscrit dans une histoire qui débute à la fin des années 1970 avec la mise en place des premières aides au retour. L'échec de cette politique a conduit le gouvernement à relancer une réflexion sur les relations entre migration, développement des régions d'origine et savoir-faire des migrants. La première moitié des années 1990 fut une période de consultation des différents acteurs concernés et d'expérimentation sur le terrain. Le PDLM, Programme de Développement Local Migrations est la première expérience en la matière. Le programme fut conduit entre 1996 et 1998 dans la région de Kayes, principale zone de départ du Mali vers la France. Il s'agissait d'un programme d'aide au montage de projet ciblant les émigrés ayant déjà effectué leur retour

14 Nair S., Rapport de bilan et d'orientation sur la politique de codéveloppement liée aux flux migratoires, Rapport au gouvernement, mission interministérielle « Migrations/codéveloppement », Paris, 1997. 
au pays et ceux désireux de le faire.

Le codéveloppement en France a donc d'abord été marqué par sa filiation avec les aides au retour. Cette filiation explique la suspicion dont cette politique est d'emblée marquée parmi les acteurs de la société civile et les chercheurs ${ }^{15}$. La critique est par ailleurs centrée sur le présupposé selon lequel le développement des zones de départ est à même de fournir une alternative à l'émigration et donc de diminuer les flux. Cette corrélation est fausse sur le court terme. A l'inverse, il est maintenant établi que le développement accélère les flux migratoires notamment en déstabilisant au niveau local les structures communautaires de production et en générant un afflux de ressources qui permet aux candidats de couvrir les coûts de l'entreprise migratoire.

En 1997, suite à l'arrivée de la gauche au pouvoir, l'universitaire Sami Nair est chargé de rédiger un rapport définissant les orientations de la politique de codéveloppement. Le rapport lui-même reste prudent. Il affirme d'emblée que le codéveloppement n'a pas pour objet le retour des migrants, tout en faisant de la maîtrise des flux par le développement des pays d'origine un objectif à atteindre. Selon l'auteur, la politique de codéveloppement vise à utiliser l'immigration légalement installée comme vecteur permanent d'aide au pays d'origine ; organiser les flux pour favoriser leur réinstallation dans le pays d'origine ${ }^{16}$. Par ailleurs le rapport définit une politique à multiples facettes, incluant non seulement l'administration centrale, mais également collectivités territoriales, associations, entreprises et universités. Le rapport contient donc les ferments d'une politique multiforme.

Suite aux recommandations du rapport Nair en 1997, fut créée la MICOMI (Mission Interministérielle Codéveloppement et

15 Daum C., « Développement des pays d'origine et flux migratoires : la nécessaire déconnexion ", Hommes et Migrations (1998), 58-72; Guengant J-P., "Migration internationale et développement : les nouveaux paradigmes ", Revue européenne des Migrations Internationales (1996) 12, 107-121; KhoudourCasteras D., Neither Migration nor Development: The Contradictions of French Co-development Policy, Paris: CEPII, 2009, p. 26.

16 Nair S., Rapport de bilan et d'orientation sur la politique de codéveloppement liée aux flux migratoires, Rapport au gouvernement, mission interministérielle «Migrations/codéveloppement », Paris, 1997, p. 5. 
Migrations Internationales) chargée de mettre en place les dispositifs de cette politique.

Toutefois, dès 1998, son action fut perturbée lorsque le gouvernement chargea l'organisme d'accompagner le retour des déboutés de la vague de régularisation qui eut lieu cette même année. Elle fut dès lors durablement discréditée aux yeux de ses partenaires tant associatifs que publics. Cette institution fut supprimée lors de l'alternance politique. En parallèle, une seconde série de consultations furent conduites, incluant cette fois les représentants des associations de migrants. Ces discussions débouchèrent sur la création, en 2001, du Forim, une plateforme associative d'ONG de migrants, officiellement appelées OSIM (Organisations de Solidarité Internationale issue des Migrations), chargée de représenter ces organisations de migrants auprès de leurs partenaires publics et privés.

En 2002, le nouveau gouvernement remplaça la MICOMI par un ambassadeur du codéveloppement, chargé de négocier la mise en place de nouveaux dispositifs avec les pays d'origine. Rattaché administrativement au secrétariat général du Ministère des Affaires Etrangères, il a un rôle de conception, de coordination entre les différents services ministériels en charge de la politique migratoire et de coopération, de dialogue avec les associations de migrants; la mise en œuvre des projets. En 2003, le gouvernement fixe les grandes lignes de cette politique. Elle est structurée autour de trois axes :

- l'appui aux projets de développement initiés par les migrants dans leur pays d'origine, avec ou sans retour de la part des concernés : Programme Migration et Initiative Economique ; aides au retour et à la réinstallation qui s'élèvent à 3500 euros pour les aides au retour volontaire et 7000 euros pour les aides au retour humanitaire ${ }^{17}$.

- la mobilisation des compétences des migrants qualifiés : le programme Interpares permet la création de doubles chaires dans les pays de départ et d'installation, programme TOKTEN élaboré par le PNUD.

- la mobilisation de l'épargne des migrants en facilitant les transferts monétaires : création d'un site internet ${ }^{18}$ proposant des informations

17 Voir sur Internet: (http://www.ofii.fr/retourner_dans_son_pays_57/aides_au_ retour_et_a_la_reinsertion_489.html).

18 Voir le site Internet (www.envoidargent.fr) qui s'est construit sur le modèle du 
pour transférer de l'argent au meilleur coût.

La création du nouveau Ministère de l'Immigration, de l'Identité Nationale et du Codéveloppement par Nicolas Sarkozy en 2007 est le dernier volet de cette évolution de la politique française de codéveloppement. Le nouveau Ministère conserve les grandes lignes adoptées par le précédent gouvernement. Deux nouveaux dispositifs furent mise en place : le permis « Compétences et Talents » encadrant l'immigration temporaire de travailleurs qualifiés; les comptes épargne codéveloppement qui ouvrent à leur détenteurs des facilités de création d'entreprise dans le pays d'origine. Ces comptes ne sont pas encore disponibles dans les établissements bancaires français.

Au cours de la dernière décennie, la confusion entre coopération au développement et politique migratoire n'a cessé de s'accentuer. La fusion au sein d'un même Ministère des services d'immigration et de codéveloppement est le couronnement d'une tendance présente dès 1997. D'une part, le codéveloppement est devenu un volet accompagnant chaque dimension de la politique migratoire, que ce soit l'objectif de sélection de main-d'œuvre qualifiée (carte Compétence et Talents) ou celle de la reconduite à la frontière des immigrés sans-papiers (aides au retour et à la réinsertion). D'autre part, le codéveloppement est inscrit dans une stratégie d'externalisation des contrôles par le biais des " accords de gestion concertée des flux migratoires et de codéveloppement ». Ces accords permettent aux signataires de bénéficier de certains dispositifs de codéveloppement en échange de leur coopération dans la lutte contre l'émigration irrégulière. Huit pays les ont aujourd'hui signés, tous Africains (Sénégal, Maurice, Gabon, Congo, Cap Vert, Bénin, Tunisie et Burkina Faso). Le terme codéveloppement a été récemment remplacé par développement solidaire dans la dénomination officielle $\mathrm{du}$ Ministère. Le Ministère soutient désormais une série de projets ayant un effet indirect sur le contrôle des flux migratoires, même si les dits projets n'incluent par directement de migrant. Le Ministère soutient par exemple un projet de modernisation de l'Etat Congolais qui vise à améliorer l'enregistrement civil des ressortissants 
congolais $^{19}$.

Ce panorama reste incomplet. La politique de codéveloppement, mobilise par ailleurs une multitude d'acteurs. Plusieurs autres programmes conduits par l'Agence Française de Développement ou la Mission de la Coopération Non Gouvernementale (MCNG) du Ministère des Affaires Etrangères également incluent des partenaires migrants. Par exemple, le Programme Concerté Maroc ${ }^{20}$ soutenu par la MCNG finance des programmes portés conjointement par une association basée en France (issus de l'immigration ou non) et une association basée au Maroc dans le but de consolider des relations associatives transnationales. Le Fonds Solidarité Prioritaire codéveloppement est un autre programme ciblant le Sénégal et le Mali. Outre le Ministère des Affaires étrangères, il faut souligner l'engagement de certaines collectivités territoriales sur cette question. La deuxième moitié des années 1990 fut également marquée par plusieurs initiatives de coopération décentralisée impliquant des populations migrantes : entre le Nord-Pas-de-Calais et Saint Louis du Sénégal, entre la Seine Saint Denis et Figuig au Maroc, entre Montreuil et la région de Kayes, ou, plus récemment, la ville de Paris. La municipalité de Paris lance en 2003 une politique destinée à soutenir les associations de migrants établies sur le territoire de la ville sur le modèle des politiques mises en œuvre au Sud de l'Europe (voir ci-dessous). Quant au FORIM, il gère une ligne de crédit destinée à soutenir les projets des ONG de migrants en France, le PRA OSIM (Programme d'Appui aux OSIM), qui s'élève à 120000 euros en 2009.

Les remaniements institutionnels incessants et la multiplicité des acteurs publics présents (ministères, agences de coopération, collectivités locales) contribuent à rendre cette politique particulièrement illisible. Quant à mesurer l'impact des plans mis en œuvre et les fonds mobilisés pour soutenir les associations de migrants, ce fut la mission confiée à l'équipe de Catherine Tasca, Sénatrice PS membre de la commission Affaires étrangères, défense et forces armées. Le rapport lui-même s'est montré très critique sur les

19 Voir sur Internet : (http://www.immigration.gouv.fr/spip.php?page= dossiers them_dev\&numrubrique $=327)$

20 Voir le site Internet (www.pcm.ma). 
résultats obtenus au regard des fonds alloués. En l'état actuel, elle reste une politique à l'état de « prototype », « enfermée dans un réduit $\mathrm{du}$ Ministère de l'immigration $»^{21}$. Au niveau du Ministère de l'immigration, le budget codéveloppement s'élevait, en 2008, à 29 millions d'euros, soit $4,5 \%$ du total consacré à la politique migratoire $^{22}$. Ce chiffre démontre à lui seul le décalage entre la rhétorique du codéveloppement et la réalité d'une politique qui reste subordonnée au contrôle des flux (près de 30000 reconduites à la frontière en 2008) et à la sélection des compétences.

En accord avec cet état semi-latent dans laquelle elle est maintenue, la politique de codéveloppement n'a eu en définitive que très peu d'effet tant sur le développement que sur les flux migratoires. L'impact le plus significatif est visible sur la société civile migrante basée en France. Jusque dans les années 1990, l'espace public républicain a interdit toute visibilité des associations de migrants. Le principe d'égalité républicaine défini pendant la Révolution Française s'oppose à toute représentation collective des personnes sur la base de l'appartenance ethnique. La thématique du développement a permis à ces organisations de dépasser les limites communautaires dans lesquelles elles ont émergé. Les organisations africaines sont les premières à en avoir bénéficié. La politique de codéveloppement française a avant tout ciblé les populations d'Afrique du Nord et de l'Ouest. En conséquence, les organisations Maliennes, Sénégalaises, Mauritaniennes et Marocaines constituent les ONG les plus nombreuses, les plus importantes et les plus actives dans le champ de la coopération ${ }^{23}$. Le Réseau des Associations des Organisations du Bassin du Fleuve Sénégal regroupe à lui seul 400 associations de migrants issus des trois pays du Bassin. Immigration Développement Démocratie est une plateforme fédérant quant à elle 24 associations

21 Tasca et al., 2007, p. 49.

22 Khoudour-Casteras D., Neither Migration nor Development: The Contradictions of French Co-development Policy, Paris: CEPII, 2009, p. 10.

23 Pour une analyse des activités des organisations maliennes et marocaines, le lecteur pourra se reporter, respectivement aux ouvrages de Daum C., Les associations de Maliens en France. Migrations, développement et citoyenneté, Paris: Karthala, 1998 ; et Lacroix T., Les réseaux marocains $d u$ développement : géographie du transnational et politique du territorial. Paris: Presses de Sciences Po., 2005. 
marocaines. La grande majorité des ONG de migrants travaillent en milieu rural, en lien avec les villages d'origine des adhérents. Leurs activités sont avant tout axées sur le soutien de l'équipement collectif et social. Les initiatives dans le domaine économique restent minoritaires. A l'instar de ce qui existe en Grande-Bretagne, les principales $\mathrm{ONG}$ de migrants opèrent à la fois au niveau politique et sur le terrain des pays du Sud. Toutefois, les activités de lobbying en France sont essentiellement conduites en collaboration avec des ONG nationales telles que le CCFD (Comité Contre la Faim et pour le Développement), la CIMADE ou l'Institut Panos. Il faut y voir une conséquence des structures de l'espace public en France qui reste moins ouvert qu'outre-manche aux organisations de type communautaire.

\subsection{Espagne et Italie : codéveloppement et coopération décentralisée}

L'Espagne et l'Italie sont deux pays d'immigration à la fois récente et massive. Tous deux ont attiré des flux en provenance du Maghreb (Maroc, Tunisie), d'Afrique de l'Ouest (Mali, Sénégal), d'Europe de l'Est (Roumanie, Ukraine, Albanie, la Moldavie) et, pour ce qui concerne l'Espagne, d'Amérique Latine (Equateur, République Dominicaine, Colombie). Dans un contexte de croissance et de besoin de main-d'œuvre, notamment dans l'agriculture, la construction ou le travail domestique, ces pays ont géré les flux d'immigration en alternant mesures restrictives et campagnes de régularisation.

Contrairement à la France et à la Grande-Bretagne, les deux pays méditerranéens connaissent un haut degré de décentralisation des structures politiques. Dans les deux cas, le codéveloppement est étroitement lié à la coopération décentralisée. Pour ces deux pays, il faut donc parler de deux types de codéveloppement qui se distinguent nettement, le codéveloppement mis en œuvre par les autorités locales et celui défini par le pouvoir central.

En Espagne, les acteurs impliqués dans le codéveloppement au niveau local sont extrêmement diversifiés, incluant municipalités (par exemple Madrid) et régions autonomes (Communautés de Madrid Valence, Andalousie, Catalogne, Pays Basque, Baléares, Murcie). A cela il faut ajouter des ONG nationales (notamment liées à l'Eglise 
ou aux syndicats) et de migrants développant leurs propres programmes $^{24}$. Le point commun de ces politiques de codéveloppement est d'insister sur la dynamique d'intégration que suscitent les opérations de codéveloppement dans la localité. Les régions misent sur la dimension intégratrice du codéveloppement tout en instrumentalisant cette politique pour développer leur compétence en matière de relations internationales (c'est particulièrement vrai pour la Catalogne et le Pays Basque). Le codéveloppement s'insère donc dans une stratégie de positionnement vis-à-vis de l'Etat central ${ }^{25}$. Un exemple est celui du Fons Catalá de Cooperacio ${ }^{26}$. Cette organisation créée en 1986 pour dynamiser la coopération entre les municipalités de la Catalogne a intégré le codéveloppement comme l'un de ces axes de travail à la fin des années 1990. La politique du Fons Català encourage la construction d'une nouvelle citoyenneté. Pour réaliser cela, il collabore avec différentes associations/institutions tant en Catalogne que dans les pays d'origine : associations d'immigrés, ONG locales, municipalités. Le Fons encourage la mise en place de réseaux d'information et de formation, un effort d'accompagnement et de «capacity-building » à destination des associations de migrants du territoire afin d'encourager leur participation aux Conseils de Coopération Municipale et aux réseaux de codéveloppement. Le Fons Català enfin est actuellement engagé dans un projet pilote sur la canalisation des envois de fonds au Sénégal financé par l'Agence Espagnole de Coopération au Développement (AECI), l'Association Espagnole des Banques et la Confédération Espagnole des Caisses d'Estalvis ${ }^{27}$.

En Italie, en 2006, il y avait une soixantaine d'opérations transrégionales et translocales en cours en Italie ${ }^{28}$. L'origine de la

24 Romero C.G., Martinez J.M., Garcia M.F., Maisonave A.C., El codesarrollo en España. Protagonistas, discursos y experiencias, Madrid: Los Libros de la 25 Id. Catarata, 2006, 256p.

26 Voir le site Internet (www.fonscatala.org).

27 Mezzetti P., Ferro A., «Politiche municipali per il cosviluppo. Esperienze europee a confronto e benchmarking del Bando sul cosviluppo del commune di Milano, 2007-2008 », CESPI Working Papers, Rome: CESPI, 2008, p. 35.

28 De Haas H., Engaging Diasporas. How Governments and Development Agencies 
politique de codéveloppement en Italie est associée avec les activités de l'Organisation des Migrations Internationales dans le pays. Cette politique a donc d'abord était marquée par l'objectif de retour des migrants dans leur pays d'origine. L'un des premiers dispositifs de codéveloppement a impliqué la région d'Emilie Romagne et l'OIM. Le programme MIDA (Migration et Développement en Afrique) fut lancé en 2001 en collaboration avec l'Organisation de l'Union Africaine. Il est destiné à encourager le transfert de compétence et faciliter le réinvestissement du capital humain dans les pays d'origine. MIDA Italie a été (modestement) financé à hauteur de 520000 euros. Il a pour cible les populations d'Afrique de l'Ouest. Paradoxalement, la réussite du programme est liée au degré d'intégration des tissus associatifs des communautés ghanéennes et sénégalaises dans la vie publique locale. Ces organisations entretiennent depuis longtemps des relations à la fois avec les autorités publiques et les syndicats. Ainsi, en Italie, la présence de Sénégalais et Ghanéens à la CGIL (syndicat italien impliqué dans la préparation des dispositifs de codéveloppement en Emilie Romagne), a facilité le soutien de projets dans les deux pays concernés par le programme MIDA. A l'inverse, une opération menée avec la communauté éthiopienne a échoué en raison du manque d'intérêt de la communauté installée en Italie et des autorités éthiopiennes ${ }^{29}$.

Depuis le milieu des années 2000, on voit apparaitre de nouvelles politiques de codéveloppement orientés non pas par des objectifs de retour mais d'intégration. A Milan, une politique de codéveloppement mise en place en 2008 a débouché sur une consultation avec les principales organisations de migrants de la ville ${ }^{30}$. L'un des objectifs majeurs du dispositif est de valoriser l'ancrage des migrants sur leur territoire d'accueil. De fait, les projets de développement sélectionnés pour être cofinancés par la ville de Milan le sont à condition d'intégrer dans son déroulement des actions qui promeuvent le dialogue entre migrants et citoyens italiens. Il est intéressant de noter que ce type de

Can Support Diaspora Involvement in the Development of Their Origin Countries, International Migration Institute (IMI), University of Oxford, for

29 Id., p. 21.

30 Mezzetti P. et al., op. cit. 
démarche se retrouve dans d'autres villes européennes, notamment à Madrid $^{31}$, et à Paris ${ }^{32}$.

On voit donc émerger une spécificité du codéveloppement mis en œuvre par les collectivités locales méditerranéennes. Les acteurs mettent en avant les dynamiques d'intégration dont les projets de développement sont le moteur. Il en va tout autrement pour les politiques élaborées par les autorités nationales. C'est avant tout le souci de la gestion des flux qui prime. En Espagne comme en Italie, l'administration centrale perçoit le codéveloppement avant tout comme un instrument de maîtrise des flux, de développement des régions d'origine et d'aide au retour. Les mesures sont orientées vers les pays de départ (retour, utilisation rationnelle des transferts d'argent, renforcement de l'investissement productif). Un exemple est le programme GRECO en Espagne, mis en œuvre par le Ministère de l'Intérieur ${ }^{33}$. Comme en France, l'intégration est une thématique absente des dispositifs au niveau national.

\section{Synthèse : les multiples échelles du codéveloppement et leur impact sur les organisations de migrants}

Les politiques de codéveloppement sont de trois ordres: accompagnement des retours volontaires, amélioration des transferts (financier, de compétences et investissements productifs), et soutien des organisations de migrants. Ce dernier champ est essentiellement constitué de mesures visant à l'amélioration du tissu associatif, au soutien des projets de développement et la création de plateformes représentatives. Pour être complet, il faudrait distinguer au sein de chacune de ces catégories les mesures qui s'adressent aux migrants qualifiés et non-qualifiés. Ces trois types de mesure se retrouvent sous diverses formes dans tous les pays étudiés, à l'exception de la GrandeBretagne qui n'a pas mis en place d'aide au retour accompagné. Mais plus que la différence des approches d'un pays à un autre, c'est la différence des codéveloppements d'une échelle à l'autre qui retient notre attention. Les dispositifs de codéveloppement changent de

\footnotetext{
Romero C.G. et al., op. cit..

Mezzetti P. et al., op. cit.

Romero C.G. et al., op. cit.
} 
nature selon le niveau auquel ils sont mis en œuvre. Il convient de distinguer entre d'une part, le niveau local, dans le cadre de la coopération décentralisée, et au niveau national, dans un cadre de gestion des flux.

A l'échelon national, la politique de codéveloppement est fondée sur une combinaison entre développement et contrôle des flux. Dans cette optique, le soutien des projets de migrants est associé avec une obligation de retour ou encore motivé par l'idée que le développement est la clé pour contenir les flux migratoires. Le codéveloppement a acquis une réputation de "visage humanitaire d'une politique sécuritaire » qui est accréditée par les faits. Aujourd'hui, non seulement la politique de codéveloppement, mais aussi la coopération au développement est de plus en plus utilisée comme la contrepartie $\mathrm{du}$ bon vouloir des pays d'origine de participer à la lutte contre l'immigration clandestine. La substitution du terme de codéveloppement par celui de développement solidaire dans la dénomination du Ministère français de l'immigration est l'illustration la plus parlante de cette évolution. Au Sud de l'Europe, la combinaison développement/contrôle est également observable: l'Italie construit une autoroute en Libye en échange de la création de patrouilles conjointes italo-libyenne en Méditerranée ${ }^{34}$; l'Espagne construit des écoles en Gambie pour la même raison ${ }^{35}$. Cette approche du codéveloppement semble enfermée dans une impasse. Ce décalage entre discours et pratique est largement dû à la méfiance des acteurs de la migration et des Etats d'origine à l'égard de cette politique.

La Grande-Bretagne a, quant à elle, développé une démarche propre en refusant de reprendre à son compte le concept de codéveloppement. La démarche de DFID est davantage d'appréhender les interactions entre migration et développement dans leur globalité et de privilégier l'engagement du secteur privé et associatif au soutien direct. Dans cette optique, le Ministère a cherché, d'une part, à améliorer les compétences des organisations de migrants afin qu'elles puissent entrer dans le champ de la coopération, et, d'autre part, à lever les obstacles à la circulation des capitaux et à l'investissement

Le Figaro, $1^{\mathrm{er}}$ septembre 2008.

35 El Pais, 10 mars 2009. 
des migrants. Cette spécificité est à rapprocher des conditions dans lesquelles a été créée l'Agence de coopération britannique. DFID fut formellement séparée du Ministère des Affaires étrangère suite à un scandale suscité par l'utilisation des fonds de la coopération pour protéger les intérêts britanniques en Afrique ${ }^{36}$. L'indépendance formelle de la politique de coopération à l'égard des autres ministères (notamment des Affaires étrangères et de l'Intérieur) est donc beaucoup plus marquée que dans les autres pays. Toutefois, il faut également voir dans cette indépendance les raisons de l'incapacité de penser l'articulation entre codéveloppement et intégration, un domaine pris en charge par le Ministère de l'Intérieur. De surcroit, la faiblesse de la décentralisation des pouvoirs au niveau local obère une prise en charge du codéveloppement par les municipalités, et ce, alors même que les collectivités locales sont les opérateurs principaux de la politique d'intégration ${ }^{37}$. La spécificité britannique est donc largement liée à son architecture et son histoire administrative.

Pour autant, au regard du bilan de ses engagements, le Département s'est borné à produire une expertise par le financement de programmes de recherche ${ }^{38}$, par la multiplication de consultations et conférences et à soutenir la formation des cadres associatifs issus de la migration sans passer par le stade de la mise en œuvre d'action de soutien. Un constat identique peut être fait au niveau des organisations internationales $^{39}$ où l'essentiel de la recherche a été menée (Banque Mondiale, OIM, ONU, etc.), mais sans autre effet que des expérimentations ponctuelles (programme MIDA de l'OIM, TOKTEN de l'UNDP, etc.). On peut voir dans ce défaut de mise en œuvre plusieurs raisons : des conflits d'intérêt avec une gestion sécuritaire

36 Porteus T., «British Government Policy in Sub-Saharan Africa under New Labour », International Affairs 81 (2005), 281-297.

37 Solomos J., Race and Racism in Britain, Basingstoke: Palgrave-Macmillan, 2003.

38 DFID soutient le «Development Research Centre on Migration, Globalisation and Poverty », un centre de recherche de l'université de Sussex consacré aux relations entre migration et développement.

39 Pour un panorama des actions entreprises par les organisations internationales en matière de codéveloppement, voir De Haas H., Engaging Diasporas. How Governments and Development Agencies Can Support Diaspora Involvement in the Development of Their Origin Countries, International Migration Institute (IMI), University of Oxford, for OXFAM NOVIB, 2006. 
des flux, une mauvaise articulation entre recherche et action au sein des organisations publiques, désintéressement des Etats du Sud comme le montre un étude sur les Document de la stratégie de réduction de la pauvreté (DSRP) en Afrique ${ }^{40}$. Ou peut-être ce manque de volonté politique est-il lié à la conscience que le codéveloppement et les migrants ne peuvent constituer à eux seuls une réponse aux problèmes de développement des zones de départ ${ }^{41}$.

Toutefois, une approche alternative du codéveloppement prend consistance au niveau des collectivités locales. Cette approche substitue à la combinaison développement/contrôle celle de développement/intégration. Cette démarche s'inscrit dans une évolution de la gouvernance locale en Europe avec l'élargissement des prérogatives des collectivités, parfois, comme en Espagne, dans une perspective de renforcement régionaliste par rapport à l'Etat central.

$\mathrm{La}$ définition $\mathrm{du}$ codéveloppement mise en avant par les collectivités locales insiste sur la bidirectionnalité de cette politique. Les dispositifs doivent bénéficier aux territoires de départ sous forme de développement et d'accueil sous forme d'intégration. L'engagement des migrants est donc conçu comme le ferment d'une intégration fondée sur une citoyenneté qui n'est pas simplement double et juxtaposée, mais transnationale et reliée. En d'autres termes, les migrants jouent d'autant mieux leur rôle de développeur qu'ils maitrisent leur environnement dans le pays d'accueil et sont capable de mobiliser des ressources multiples. Inversement, leur intégration dans la société d'accueil profite de la reconnaissance publique de leurs engagements transnationaux.

Au-delà de cette complexité de la politique de codéveloppement, ce chapitre fait également ressortir la faiblesse des résultats sur le terrain, tant du développement que de la maitrise des flux. C'est cependant sur le plan de la visibilité des organisations de migrants, en particulier africaines, que les dispositifs publics ont produit le plus de résultat. C'est notamment le cas en Grande-

40 Black R., Migration and Pro-Poor Policy in Africa, Brighton: Development Research Centre on Migration, Globalisation and Poverty, 2004, p. 27.

41 Vaneeckhaute H., El codesarrollo, de un proceso histórico de oportunidades y desarrollo hacia una herramienta más de explotación y subdesarrollo, Pangeas, 2002. 
Bretagne et en France où des plateformes d'ONG de migrants ont été créées (respectivement le FORIM et le CfD). Mais c'est aussi le cas en Espagne et en Italie ou les liens entre associations et pouvoirs publics ont été renforcés. D'une façon générale les politiques articulant migrations et développement ont largement poussé les organisations de migrants à formaliser leurs activités de développement. Une nouvelle génération d'ONG de migrants est apparue afin de faire le lien entre les collectifs informels de porteurs de projets et les bailleurs de fonds. Nous avons cité IDD et le Réseau du Fleuve Sénégal en France, AFFORD en Grande Bretagne. Nous aurions pu évoquer les cas de la CODENAF et ATIME, deux organisations marocaines en Espagne ${ }^{42}$. On retrouve ces ONG dans les plateformes représentatives nationales ou locales. Ceci engendre parfois des attitudes clientélistes de la part des pouvoirs locaux. Ainsi, en Catalogne, la politique de codéveloppement a servi de support à une stratégie clientéliste de l'autorité régionale à l'égard des élites associatives nord africaines ${ }^{43}$. Les effets des dispositifs de codéveloppement sont donc complexes et nuancés. Pour produire une image plus complète de l'impact des politiques «migration et développement » sur le tissu associatif immigré, il serait nécessaire de resituer le codéveloppement dans les jeux politique nationaux. En France, il semble que la réappropriation municipale du codéveloppement se fait dans l'optique d'opposer un codéveloppement de gauche aux orientations sécuritaires du gouvernement. En Espagne, le codéveloppement est devenu un des pivots autours desquels se négocie la répartition des pouvoirs entre Etat et régions. En Grande-Bretagne, c'est plutôt au niveau des relations entre Ministères que cette politique se situe comme enjeu stratégique.

Enfin, il faudrait aborder le positionnement des organisations de

42 Le cas italiens diffère cependant. La faiblesse du tissu associatif immigré en Italie est à mettre sur le compte de se jeunesse, mais également de l'emprise des associations pro immigrées italiennes, notamment catholiques, qui empêchent l'émergence des associations immigrées comme interlocuteur public.

43 Ostergaard Nielsen E., "Codevelopment: Collaboration or Co-option? Transnational Civic Engagement of Moroccan Associations in Catalonia". European Dynamics of Citizenship. Public policies and migrant activities. Budapest, 2006. 
migrants vis-à-vis des Etats d'origine et de leurs politiques. Là encore ce positionnement donne lieu à des stratégies complexes, largement tributaires des conditions historiques dans lesquelles la migration s'est produite pour chacun de ces groupes. De cette double inscription, à la fois au Nord et au Sud, procède l'émergence d'une société civile migrante à l'échelle transnationale. Celle-ci doit donc être saisie dans un contexte international plus large, aux confins des phénomènes transnationaux et des relations interétatiques. 